\title{
Controle automático do espectro de gotas de pulverizador hidro- pneumático em função do déficit de pressão de vapor d'água no $\operatorname{ar}^{1}$
}

\author{
Cleyton Batista de Alvarenga ${ }^{2}$, Mauri Martins Teixeira ${ }^{3}$, \\ Sérgio Zolnier ${ }^{3}$, Robson Shigueaki Sasaki ${ }^{3}$, Paula Cristina Natalino Rinaldi ${ }^{4}$
}

\begin{abstract}
Automatic control of the droplet spectrum of a hydropneumatic sprayer according to the vapor pressure deficit in the air

The principle of fractionation and droplets size are crucial for the success of pest control. This study aimed at developing and evaluating an automated system to control the spraying droplet spectrum according to the vapor pressure deficit in the air. The automatic control system consisted of a temperature and relative humidity sensor, a microcontroller, a servomotor and a hydropneumatic sprayer. Spraying was performed on water sensitive paper labels arranged on wooden supports for different vapor pressure deficits in the air. After scanning and analysing the paper labels, three working pressures were selected $(633 \mathrm{kPa}$, $844 \mathrm{kPa}$ and $1,055 \mathrm{kPa}$ ) for use in the automated system. The results indicated that the controller efficiently maintained the droplets spectrum when spraying pesticides. Automation enabled the maintenance of the spray volume by altering the working pressure, according to the vapor pressure deficit in the air. Spraying with the automated system presented a droplet spectrum quality superior to the one obtained by using the manual system at the pressure of $633 \mathrm{kPa}$.
\end{abstract}

KEY-WORDS: Pesticide application technology; evaporation of droplets during pesticide spraying; embedded electronics in sprayers.

\section{INTRODUÇÃO}

$\mathrm{O}$ uso intensivo de agrotóxicos tem gerado crescente preocupação à sociedade, a qual, por sua vez, aumentou a pressão sobre as autoridades de planejamento agrícola e ambiental, exigindo novas maneiras de se dosar a quantidade de agrotóxicos perdida para a atmosfera, a fim de prever a contaminação

\section{RESUMO}

O princípio de fracionamento e o tamanho de gotas são fundamentais para o sucesso do controle fitossanitário. Objetivou-se, com este trabalho, desenvolver e avaliar um sistema automatizado, para controlar os parâmetros do espectro de gotas da pulverização, em função do déficit de pressão de vapor d'água no ar. O sistema de controle automático constituiu-se de um sensor de temperatura e umidade relativa, um microcontrolador, um servomotor e um pulverizador hidropneumático. A pulverização foi realizada em etiquetas de papel hidrossensível, dispostas em suportes de madeira, para diferentes déficits de pressão de vapor d'água no ar. Posteriormente à digitalização e análise das etiquetas, foram selecionadas três pressões de trabalho $(633 \mathrm{kPa}, 844 \mathrm{kPa}$ e $1.055 \mathrm{kPa}$ ), para serem utilizadas no sistema automatizado. Os resultados indicaram que o controlador foi eficiente na manutenção do espectro de gotas da pulverização de agrotóxicos. A automatização permitiu a manutenção do volume de calda, alterando a pressão de trabalho, em função do déficit de pressão de vapor d'água no ar. A pulverização com o sistema automatizado apresentou qualidade do espectro de gotas superior ao sistema manual, à pressão de $633 \mathrm{kPa}$.

PALAVRAS-CHAVE: Tecnologia de aplicação de agrotóxicos; evaporação de gotas na pulverização de agrotóxicos; eletrônica embarcada em pulverizadores.

ambiental e os riscos a trabalhadores, consumidores e animais (Gil et al. 2008, Yu et al. 2009).

A aplicação de agrotóxicos é tarefa complexa, que envolve uma série de fatores, como a grande diversidade de equipamentos e métodos de pulverização, diferenças entre produtos químicos, diversidade de culturas e hábitos de crescimento, número de insetos e doenças, disparidades na habilidade

1. Trabalho recebido em jun./2012 e aceito para publicação em fev./2013 ( ${ }^{\circ}$ registro: PAT 19086).

2. Universidade Federal de Mato Grosso (UFMT), Instituto de Ciências Agrárias e Ambientais, Sinop, MT, Brasil.

E-mail: cleytonalvarenga@cpd.ufmt.br.

3. Universidade Federal de Viçosa (UFV), Instituto de Ciências Agrárias, Viçosa, MG, Brasil. E-mails: mauri@ufv.br, zolnier@ufv.br, robsonsasaki@yahoo.com.br.

4. Instituto Federal de Educação, Ciência e Tecnologia do Sudeste de Minas Gerais (IFSudesteMG), Rio Pomba, MG, Brasil. E-mail: paula.rinaldi@ifsudestemg.edu.br. 
dos operadores, condições meteorológicas incontroláveis, segurança do trabalhador, regulamentos e legislações ambientais, volume de calda, tamanho da copa e relação custo-benefício das aplicações de agrotóxicos (Llorens et al. 2010, Xu et al. 2010, Jeon et al. 2011).

A tecnologia de informação é uma ferramente com potencial para auxiliar na determinação do tamanho das gotas, na pulverização de agrotóxicos. Segundo Díaz et al. (2011), nos últimos anos, as redes de sensores sem fio têm surgido como uma tecnologia promissora, na área de sistemas embarcados, sendo compostas por dispositivos de pequeno porte, normalmente ligados por conexões sem fios, com capacidade de transmitir dados com alta velocidade de processamento. Neste sentido, a agricultura é um ambiente ideal para aplicar esta tecnologia, devido à sua sensibilidade aos fatores meteorológicos.

A padronização da eletrônica embarcada em máquinas agrícolas é motivo de preocupação mundial, sendo a norma ISO 11783 o resultado deste esforço. A norma é composta por um conjunto de definições, regras e procedimentos, que têm por objetivo permitir a conexão e a troca de informações entre unidades de controle de um trator e os implementos agrícolas, sendo uma tentativa para se aplicar o conceito de plug and play entre máquinas e implementos agrícolas cada vez mais informatizados.

Com relação à precisão das leituras, uma alternativa para se evitar erros é a utilização de sensores digitais. O sistema digital oferece muitas vantagens, em relação ao sistema analógico, pois um sinal digital é menos sensível a interferências eletromagnéticas e, além disto, o protocolo de comunicação estabelece mecanismos que permitem detectar e corrigir erros de transmissão (Silva et al. 2007).

O panorama, no Brasil e no mundo, indica uma trajetória na qual a agricultura se tornará cada dia mais automatizada, nas diferentes etapas de execução do trabalho. A automatização poderá contribuir para elevar a qualidade e o controle das aplicações, bem como para a sustentabilidade ambiental e econômica do processo produtivo, impulsionando o desenvolvimento econômico e social do Brasil.

A aplicação de agrotóxicos deveria ser feita pela manhã e ao final do dia, objetivando reduzir o efeito dos fatores meteorológicos, como temperatura e umidade relativa do ar. Em determinadas situações, os produtores têm um intervalo entre aplicações bastante pequeno, em decorrência de altas infestações de insetos, patógenos ou plantas daninhas, frequência das chuvas e temperaturas elevadas, dentre outros aspectos. Neste sentido, um pulverizador dotado de sistema de controle automático do espectro de gotas e demais parâmetros da pulverização, que leve em conta as variações meteorológicas, possibilitará maior eficiência e qualidade das aplicações.

Assim, objetivou-se, com este trabalho, desenvolver um sistema automático para controlar o espectro de gotas produzidas por um pulverizador hidropneumático, em função do déficit de pressão de vapor d'água no ar.

\section{MATERIAL E MÉTODOS}

O experimento foi desenvolvido no Laboratório de Aplicação de Agrotóxicos e em área experimental do Departamento de Fitotecnia da Universidade Federal de Viçosa (MG), durante o mês de setembro de 2011.

O sistema para a automatização do pulverizador foi constituído por um sensor de temperatura e umidade relativa, um microcontrolador e um servomotor. Este sistema foi adaptado a um pulverizador hidropneumático, marca Hatsuta, modelo SS-420, equipado com bomba hidráulica de pistão, modelo S60, e um ventilador axial de $700 \mathrm{~mm}$ de diâmetro. Inicialmente, este equipamento foi acoplado, em condições de laboratório, a um trator John Deere, modelo 5705, com potência nominal de $62,5 \mathrm{~kW}$ $(85 \mathrm{cv})$. Posteriormente, no ensaio de campo, foi acoplado a um trator New Holland, modelo TL75E, com potência de $55,2 \mathrm{~kW}(75 \mathrm{cv})$.

A temperatura e a umidade relativa do ar foram medidas por um sensor conjugado, modelo Humitter 50Y, fabricado pela Vaisala Inc., em Woburn, MA, USA, e alimentado pela bateria de $12 \mathrm{~V}$ do trator. A escala de medição do sensor, para umidade relativa, é de $0 \%$ a $100 \%$, com precisão de $\pm 3 \%$, e, para a temperatura, de $-40^{\circ} \mathrm{C}$ a $60^{\circ} \mathrm{C}$, com precisão de $\pm 0,6^{\circ} \mathrm{C}$ (à temperatura de $20^{\circ} \mathrm{C}$ ). Este sensor já foi utilizado para monitorar as condições meteorológicas em lavoura de café (Pezzopane et al. 2010).

A tensão de funcionamento das entradas e saídas do sensor variou de 7 Vcc a 28 Vcc, com corrente de 2,0 miliampéres. A temperatura e a umidade relativa correspondem a um sinal de tensão do sensor, que, após ser convertido, com o auxílio do microcontrolador, enviou, como sinal de saída, a umidade e a temperatura propriamente ditas ao computador, 
segundo as seguintes equações (Vaisala 2011):

$$
\begin{aligned}
& \mathrm{T}_{\mathrm{ar}}=-40+100\left[\left(\frac{5}{1023}\right) \text { sinal_ } \mathrm{T}_{\mathrm{ar}}\right] \\
& \mathrm{UR}=100\left[\left(\frac{5}{1023}\right) \text { sinal_UR }\right]
\end{aligned}
$$

em que $\mathrm{T}_{\text {ar }}=$ temperatura do ar $\left({ }^{\circ} \mathrm{C}\right)$ e $\mathrm{UR}=$ umidade relativa do ar (\%).

O Arduino Duemilanove é uma placa de microcontrolador com processador da ATMEL, modelo ATmega328, com 14 pinos de entrada e saída digital, sendo que seis podem ser utilizados como saídas analógicas Pulse-Width Modulation (PWM) e seis como entradas analógicas, um cristal oscilador de $16 \mathrm{MHz}$, uma conexão Universal Serial Bus (USB), uma entrada para alimentação e um botão de reset. O Arduino pode ser alimentado pela conexão USB ou por qualquer fonte de alimentação externa com tensão de 6-20 volts, sendo a alimentação recomendada de 7-12 volts, com corrente contínua de 40-50 miliampéres (Arduino 2011).

A unidade de processamento foi responsável pelo controle do sistema automatizado. A partir desta unidade, saíram as conexões para os comandos de gerenciamento do sistema de controle da pressão. O microcontrolador processou as informações provenientes dos sensores de temperatura e umidade relativa, acionando o servomotor. As informações para o funcionamento do registro de pressão foram processadas no microcontrolador e, por meio de um algoritmo implementado em linguagem $\mathrm{C}^{++}$, foi determinado o ângulo de giro do motor, para alterar a pressão de trabalho, em função do déficit de pressão de vapor d'água no ar.

O servomotor atuou como mecanismo controlador da pressão de trabalho e foi alimentado por por uma bateria de 9 V. O servomotor utilizado foi um Turnigy, modelo S8166M de $154 \mathrm{~g}$, com torque de $33 \mathrm{kgf} \mathrm{cm}^{-2}$. O sensor, juntamente com o microcontrolador, foi acondicionado em uma caixa para componentes eletrônicos, constituindo a unidade de processamento.

Posteriormente, os componentes foram colocados em um abrigo meteorológico embarcado no trator, juntamente com um computador, para armazenamento dos dados. A alimentação do computador foi realizada pela bateria do trator. Para isto, utilizou-se um inversor de corrente, modelo Portawattz 300. Os dispositivos que constituíram o sistema de automação foram ligados utilizando-se o sistema plug and play, conforme estabelecido na norma ISO 11783.
A aplicação foi realizada sobre etiquetas de papel hidrossensível, dispostas em suportes de madeira, utilizando-se a ponta de jato cônico vazio JA-2, fabricada pela Jacto, em diferentes déficits de pressão de vapor d'água no ar.

Durante os ensaios, ajustaram-se as pressões, manualmente, até a obtenção dos valores de $633 \mathrm{kPa}, 703 \mathrm{kPa}, 774 \mathrm{kPa}, 844 \mathrm{kPa}, 914 \mathrm{kPa}$, $985 \mathrm{kPa}, 1.055 \mathrm{kPa}, 1.125 \mathrm{kPa}, 1.195 \mathrm{kPa}, 1.266 \mathrm{kPa}$, $1.336 \mathrm{kPa}, 1.406 \mathrm{kPa}$ e $1.477 \mathrm{kPa}$, recomendados pelo fabricante da ponta. Posteriormente, as etiquetas foram digitalizadas e analisadas e selecionadas as pressões de $633 \mathrm{kPa}, 844 \mathrm{kPa}$ e $1.055 \mathrm{kPa}$, para serem utilizadas no sistema automatizado.

A pulverização sobre os suportes de madeira foi realizada à velocidade de $9 \mathrm{~km} \mathrm{~h}^{-1}$, pois esta permitiu utilizar as etiquetas hidrossensíveis para a mensuração do espectro de gotas sem que houvesse molhamento total das etiquetas.

$\mathrm{O}$ experimento foi repetido em um laranjal da variedade Pêra Rio, à pressão de $1.055 \mathrm{kPa}$, com déficit de pressão de vapor d'água no ar variando de $0,76 \mathrm{kPa}$ a $0,86 \mathrm{kPa}$, sendo empregados o sistema manual e o automatizado, à velocidade de $2,8 \mathrm{~km} \mathrm{~h}^{-1}$, e pulverizado volume de calda igual a $696 \mathrm{~L} \mathrm{ha}^{-1}$.

Os critérios para a seleção das pressões para a automação do pulverizador foram os parâmetros da pulverização obtidos nos ensaios realizados no laboratório, a partir da análise das etiquetas dispostas nos suportes de madeira e em analisador de partículas a laser (Spraytech, Malvern Instruments Co.), visando ao tratamento fitossanitário. Nestes ensaios, foram considerados os parâmetros densidade de gotas, diâmetro da mediana volumétrica, percentagem de cobertura, $\mathrm{Dv}_{0,9}, \mathrm{Dv}_{0,1}$, coeficiente de homogeneidade e amplitude relativa, com o posicionamento das etiquetas amostradoras à altitude de $0,5 \mathrm{~m}, 1,5 \mathrm{~m}$ e $2,5 \mathrm{~m}$, em relação ao solo, em diferentes posições (Figura 1).

O espectro de gotas também foi obtido utilizando-se analisador de partículas a laser, que se baseia na difração da trajetória da luz ao chocar com as gotas. Este equipamento é dotado de lente focal de $750 \mathrm{~mm}$. A aplicação foi feita de modo que o jorro atingisse, transversalmente, o feixe luminoso, permitindo a obtenção direta do espectro de gotas. A ponta foi posicionada a $0,40 \mathrm{~m}$ do feixe óptico. No ensaio, utilizou-se água pura, conforme proposto por Câmara et al. (2008) e Cunha et al. (2010). O analisador foi calibrado para contabilizar gotas na 


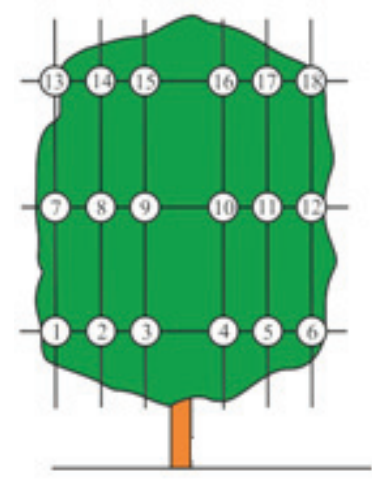

Figura 1. Pontos de posicionamento das etiquetas amostradoras hidrossensíveis e de amostragem de folhas no dossel da planta (Viçosa, MG, 2011).

classe de tamanho de 0,10-2.500 $\mu \mathrm{m}$ e, assim, obter um rigoroso espectro de gotas.

As cores verde, azul e vermelho representavam, respectivamente, as pressões de $633 \mathrm{kPa}$, $844 \mathrm{kPa}$ e $1.055 \mathrm{kPa}$, selecionadas para diferentes déficits de pressão de vapor d'água no ar. As condições para esta avaliação foram o tempo gasto para que o servomotor alterasse a pressão de $633 \mathrm{kPa}$ para $844 \mathrm{kPa}$ e de $844 \mathrm{kPa}$ para $633 \mathrm{kPa}$, bem como o tempo requerido para que a pressão fosse alterada de $844 \mathrm{kPa}$ para $1.055 \mathrm{kPa}$ e de $1.055 \mathrm{kPa}$ para $844 \mathrm{kPa}$. Foram realizadas dez repetições, em cada condição, e o tempo médio foi apresentado juntamente com o desvio-padrão.

Para simular a alteração do déficit de pressão de vapor d'água no ar, utilizou-se um aquecedor elétrico da marca Mallory, a fim de aumentar a temperatura do ambiente em torno do sensor e, consequentemente, aumentar o déficit de pressão. O déficit de pressão de vapor foi calculado a partir dos valores de temperatura do ar e dos valores correspondentes da pressão parcial e de saturação de vapor da água no ar. A pressão de saturação de vapor da água foi determinada utilizando-se a equação proposta por Tetens (1930):

$$
e_{5}=0,610810^{\left[\frac{(7,5 \mathrm{~T})}{(237,5+\mathrm{T})}\right]}
$$

em que $e_{s}=$ pressão de saturação do vapor d'água $(\mathrm{kPa})$ e $\mathrm{T}=$ temperatura do ar ambiente $\left({ }^{\circ} \mathrm{C}\right)$.

A partir da umidade relativa do ar, determinou-se a pressão parcial de vapor d'água, utilizando-se a equação

$$
\mathrm{e}_{\mathrm{a}}=\frac{{\mathrm{UR} \mathrm{e}_{5}}_{100}}{100}
$$

em que e $e_{\mathrm{a}}=$ pressão parcial de vapor d'água $(\mathrm{kPa})$ e $\mathrm{UR}=$ umidade relativa do ar (\%).

Posteriormente, utilizando-se a pressão de vapor de saturação e a pressão parcial de vapor d'água, calculou-se o déficit de pressão de vapor d'água, que é a medida da capacidade de evaporação do líquido para o ambiente, no instante em que foram realizadas as pulverizações, controlando-se a abertura e o fechamento do registro de pressão e o retorno da calda para o tanque, segundo a equação DPV $=\mathrm{e}_{\mathrm{s}}-\mathrm{e}_{\mathrm{a}}$, em que DPV = déficit de pressão de vapor d'água no ar $(\mathrm{kPa})$.

As aplicações foram realizadas com ajustes manuais de pressão e com o sistema automatizado, nos períodos do dia que apresentaram as condições de déficit de pressão desejadas, sob diferentes condições meteorológicas, visando à obtenção de pulverização com qualidade técnica e segurança, do ponto de vista ambiental, para possibilitar boa eficiência de aplicação. Para facilitar a visualização da condição em que se encontrava o sistema de automatização, foram adicionados LEDs de diferentes cores, cada um correspondendo a um déficit de pressão (Tabela 1).

Após os testes com etiquetas hidrossensíveis, nos suportes de madeira, a fim de facilitar a comunicação com o operador da máquina e a tomada de decisão no campo, foi acoplado ao painel do trator um visor de LCD (Liquid Crystal Display), para informar a velocidade do trator e o déficit de pressão de vapor, visando a manter o volume de calda constante, após a alteração da pressão de trabalho.

\begin{tabular}{|c|c|c|c|c|}
\hline \multirow{2}{*}{ Tratamentos } & Déficit de pressão & Pressão de trabalho & Vazão & \multirow{2}{*}{ LEDs } \\
\hline & $\mathrm{kPa}$ & & $\mathrm{L} \mathrm{min}^{-1}$ & \\
\hline DPV 1 & $0,0<\mathrm{DPV}<1,0 \pm 0,05$ & 633 & 0,77 & verde \\
\hline DPV 2 & $1,0<\mathrm{DPV}<2,0 \pm 0,05$ & 844 & 0,85 & azul \\
\hline DPV 3 & $2,0<\mathrm{DPV}<3,0 \pm 0,05$ & 1.055 & 0,93 & vermelho \\
\hline
\end{tabular}

Tabela 1. Condições meteorológicas para ocorrer a pulverização (Viçosa, MG, 2011).

Fonte: Dados da pesquisa. 
No instante em que as condições meteorológicas apontassem a necessidade de alteração da velocidade, a tela permanecia piscando, de modo intermitente, por dez segundos, com o intuito de alertar para a mudança de marcha. Esta interface de comunicação entre o operador e o sistema eletrônico teve como objetivo reduzir a possibilidade de erro, por parte do aplicador, e permitir que sua atenção fosse aproveitada na observação dos outros aspectos da aplicação. O sistema automatizado completo foi posicionado de maneira a facilitar o acesso do operador aos dispositivos, para possíveis ajustes e manutenções.

O programa computacional foi elaborado visando a facilitar e informar ao responsável pela pulverização o funcionamento do pulverizador e das condições psicrométricas, no momento da aplicação. Deste modo, o sensor monitorava ininterruptamente a temperatura e a umidade e, a cada trinta segundos, disponibilizava as informações detalhadas na tela do computador.

As variáveis fornecidas foram o número de entradas ou leituras, horário, temperatura do ar, umidade relativa, déficit de pressão de vapor d'água no ar, pressão de trabalho, vazão média das pontas, posição do servomotor (em graus) e a velocidade de trabalho.

Nas condições ambientais em que o déficit de pressão de vapor fosse superior a $3,0 \mathrm{kPa}$, o LCD permanecia piscando continuamente, informando ao operador que deveria interromper a pulverização. Entretanto, durante o experimento, o déficit de pressão não alcançou este patamar. A velocidade do vento foi monitorada com o auxílio de um anemômetro portátil Instruterm, modelo AD-250, não embarcado ao trator.

As velocidades do vento foram inferiores a $0,5 \mathrm{~m} \mathrm{~s}^{-1}$, durante a realização do ensaio no laboratório, considerando-se as aplicações com ajuste manual e automatizado da pressão. A temperatura, no ensaio com ajuste manual, variou entre $19^{\circ} \mathrm{C}$ e $31^{\circ} \mathrm{C}$ e a umidade relativa do ar entre $37 \%$ e $74 \%$. Nos ensaios a campo, considerando-se o déficit de pressão entre $0,0 \mathrm{kPa}$ e $1,0 \mathrm{kPa}$, a temperatura média foi de $25^{\circ} \mathrm{C}$, a umidade relativa média de $78 \%$ e o déficit de pressão variou de $0,76 \mathrm{kPa}$ a $0,86 \mathrm{kPa}$. Durante todo o experimento, as pulverizações foram realizadas com velocidade do vento abaixo de $2,0 \mathrm{~m} \mathrm{~s}^{-1}$.

As aplicações foram realizadas em delineamento de blocos casualizados e os dados analisados utilizando-se o programa SAS 9.2. Para a comparação entre as médias dos sistemas manual e automatiza- do, procedeu-se à análise de variância dos dados e aplicou-se o teste $\mathrm{F}$, a 5\%, com três repetições.

\section{RESULTADOS E DISCUSSÃO}

No ensaio realizado no laboratório, as variações psicrométricas possibilitaram a determinação do déficit de pressão de vapor d'água no ar entre $0,0 \mathrm{kPa}$ e 3,0 kPa, para os sistemas manual e automatizado. As condições psicrométricas, durante estes ensaios, permitiram a obtenção dos três déficits de pressão de vapor d'água no ar requeridos para a realização do experimento, com o sistema de automação montado no trator. A variação das condições de temperatura e umidade relativa, ao longo do dia, visando à aplicação de agrotóxicos, também foi estudada por Balan et al. (2008), que observaram, durante o dia, o mesmo comportamento obtido nesta pesquisa.

Os períodos da manhã e final da tarde foram considerados ideais para a aplicação de agrotóxicos, principalmente para o controle de insetos e patógenos, por requererem pulverizações com gotas pequenas. Deve-se destacar que, devido às proporções continentais do Brasil, o momento ideal para aplicação, considerando-se as condições psicrométricas, varia de acordo com as regiões produtoras. Diante disto, os profissionais envolvidos com a tecnologia de aplicação devem utilizar o vasto portfólio de pontas de pulverização disponível no mercado, como alternativa para se obter sucesso no controle fitossanitário.

A evaporação é um fenômeno que interfere diretamente na tomada de decisão do agricultor e/ ou operador, pois a elevação do volume de calda amplia os danos ambientais causados pelo emprego inadequado das técnicas de pulverização. A maneira mais adequada para se reduzir os efeitos da evaporação é a realização das pulverizações nas primeiras horas da manhã ou ao final da tarde, ratificando as conclusões obtidas por Balan et al. (2008) e Teske et al. (2011), bem como a seleção correta das pontas, para produzir gotas adequadas à condição de clima, durante a pulverização.

A ponta JA-2 é indicada para trabalhar na faixa de pressão de $633 \mathrm{kPa}$ a $1.477 \mathrm{kPa}$, porém, observou-se que, a partir de $1.055 \mathrm{kPa}$, o efeito da pressão no diâmetro da mediana volumétrica foi pequeno, indicando que, a partir de determinada pressão, o tamanho das gotas é pouco alterado (Figura 2). O aumento da pressão representa um aumento na vazão, maior custo energético, desgaste de pontas, rompi- 


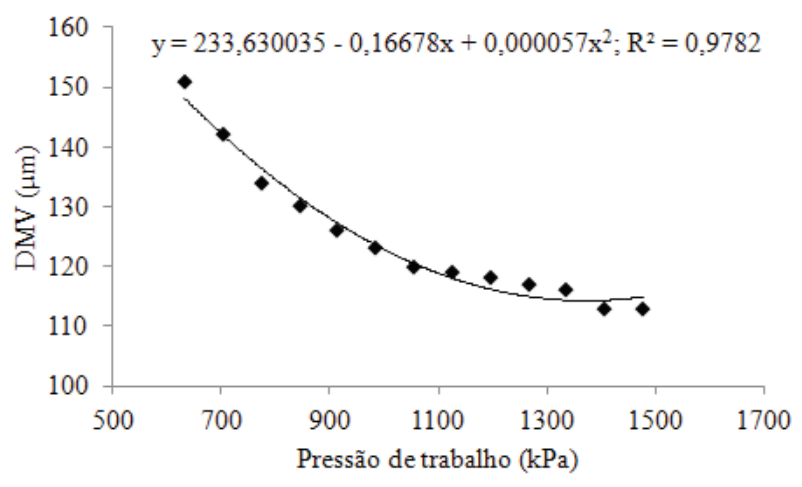

Figura 2. Diâmetro da mediana volumétrica da ponta JA-2, em função das diferentes pressões de trabalho, obtido em analisador de partículas a laser (Viçosa, MG, 2011).

mento de mangueiras, conexões e filtros e pequeno acréscimo no tamanho das gotas.

$\mathrm{Na}$ análise efetuada utilizando-se o analisador de partículas a laser, a ponta de jato cônico vazio JA-2 produziu tamanho de gotas que variou de $151 \mu \mathrm{m}$ a $109 \mu \mathrm{m}$, para as pressões de $633 \mathrm{kPa}$ a $1.477 \mathrm{kPa}$, respectivamente, proporcionando redução de $28 \%$ no tamanho das gotas. As gotas produzidas por esta ponta são susceptíveis à deriva e à evaporação, em condições meteorológicas adversas, por isto, a seleção da ponta é importante para o sucesso do controle fitossanitário.

À pressão de $633 \mathrm{kPa}$, observou-se que, para a maioria dos parâmetros, não houve diferenças entres os dois sistemas, porém, os parâmetros que evolveram o tamanho de gotas $\left(\mathrm{Dv}_{0,1}, \mathrm{Dv}_{0,5}\right.$ e $\left.\mathrm{Dv}_{0,9}\right)$ apresentaram maiores valores no sistema automatizado (Tabela 2).

Nas pressões de $844 \mathrm{kPa}$ e $1.055 \mathrm{kPa}$, não houve diferença significativa para nenhum dos parâmetros analisados entre o sistema manual e automatizado. A variação no espectro de gotas, à pressão de $633 \mathrm{kPa}$, pode ter ocorrido por diversas razões. Primeiramente, é difícil repetir duas pulverizações com espectros iguais, em etiquetas hidrossensíveis, mesmo em condições idênticas, devido à variação meteorológica, velocidade do trator, pressão de trabalho, etc. Outra razão foi o efeito do déficit de pressão de vapor, que, na época da pulverização, sem automatização da pressão, foi de $0,76 \mathrm{kPa}, 1,76 \mathrm{kPa}$ e 2,63 $\mathrm{kPa}$. Na pulverização com sistema automatizado, foi de $0,70 \mathrm{kPa}, 1,63 \mathrm{kPa}$ e 2,72 $\mathrm{kPa}$. Esta diferença pode ter aumentado o tamanho das gotas, à pressão de $633 \mathrm{kPa}$.

A capacidade de evaporação das gotas, em diferentes condições ambientais, também foi comprovada por Yu et al. (2009). Porém, o resultado mais importante foi que o sistema automatizado permitiu uma pulverização com o espectro de gotas adequado ao controle fitossanitário.

$\mathrm{Na}$ comparação entre os sistemas, pode-se afirmar que, nos ajustes manuais, as probabilidades de erros são maiores. Deste modo, corroborando Pereira (2008), a automatização do pulverizador possibilitou a redução do esforço mental e da fadiga do operador e retirou a responsabilidade da tomada de decisão, em relação ao aumento ou redução da pressão e alteração no volume de calda pulverizado. Estes resultados indicam que a automação é imprescindível à mecanização agrícola brasileira, principalmente como alternativa à falta de treinamento dos operadores.

A atenção despendida pelo operador aos fatores psicrométricos, temperatura e umidade relativa do ar foi direcionada ao adequado funcionamento do conjunto trator-pulverizador. Deste modo, o aplicador teve mais tempo para observar o entupimento de pontas e o direcionamento da calda e do ar para a copa das plantas, em função de sua altura, dentre outras variáveis que afetam a eficiência da pulverização de agrotóxicos. A atenção aos fatores meteorológicos gera preocupação ao operador e, consequentemente, pode levá-lo a negligenciar outros aspectos relevantes da pulverização, corroborando os estudos de Scarlett (2001).

A pulverização, no campo, também foi utilizada para comparar os sistemas manual e automatizado, a fim de constatar o funcionamento da automação e

Tabela 2. Efeito do sistema de aplicação nos parâmetros da pulverização, à pressão de 633 kPa (Viçosa, MG, 2011).

\begin{tabular}{|c|c|c|c|c|c|c|c|}
\hline \multirow{2}{*}{ Sistema } & \multirow{2}{*}{$\mathrm{DMV}^{(1)}$} & \multirow{2}{*}{$\mathrm{CH}^{(2)}$} & \multirow{2}{*}{$\mathrm{COB}^{(3)}$} & \multirow{2}{*}{$\mathrm{POP}^{(4)}$} & $\mathrm{Dv}_{0,9}$ & $\mathrm{Dv}_{0,1}$ & \multirow{2}{*}{$\operatorname{Span}^{(5)}$} \\
\hline & & & & & \multicolumn{2}{|c|}{$-\mu \mathrm{m}-$} & \\
\hline Manual & $456 b$ & $3,3 \mathrm{a}$ & $27 \mathrm{a}$ & $188 \mathrm{a}$ & $667 b$ & $137 \mathrm{~b}$ & $1,1 \mathrm{a}$ \\
\hline Automatizado & $550 \mathrm{a}$ & $4,6 \mathrm{a}$ & $36 \mathrm{a}$ & $195 \mathrm{a}$ & $861 \mathrm{a}$ & $179 \mathrm{a}$ & $1,2 \mathrm{a}$ \\
\hline C. V. $(\%)$ & 14,48 & 43,81 & 43,50 & 32,80 & 19,61 & 21,14 & 16,61 \\
\hline
\end{tabular}

${ }^{(1)}$ Diâmetro da mediana volumétrica $(\mu \mathrm{m}) ;{ }^{(2)}$ Coeficiente de homogeneidade; ${ }^{(3)}$ Percentagem de cobertura (\%); ${ }^{(4)}$ População de gotas $\left(\right.$ gotas cm $\left.{ }^{-2}\right) ;{ }^{(5)}$ Amplitude relativa. Médias seguidas de letras iguais, na coluna, não diferem entre si, pelo teste Tukey, a $5 \%$. 
Tabela 3. Efeito do sistema de aplicação nos parâmetros da pulverização realizada no campo (Viçosa, MG, 2011).

\begin{tabular}{|c|c|c|c|c|c|c|c|c|}
\hline \multirow{2}{*}{ Sistema } & \multirow{2}{*}{$\mathrm{DMV}^{(1)}$} & \multirow{2}{*}{$\mathrm{CH}^{(2)}$} & \multirow{2}{*}{$\mathrm{COB}^{(3)}$} & \multirow{2}{*}{$\mathrm{POP}^{(4)}$} & $\mathrm{Dv}_{0,9}$ & $\mathrm{Dv}_{0,1}$ & \multirow{2}{*}{$\operatorname{Span}^{(5)}$} & \multirow{2}{*}{$\mathrm{DEP}^{(6}$} \\
\hline & & & & & \multicolumn{2}{|c|}{$\longrightarrow \mu \mathrm{m}-$} & & \\
\hline Manual & $324 a$ & $5,4 a$ & $23 a$ & $249 a$ & $605 a$ & $129 \mathrm{a}$ & $1,5 \mathrm{a}$ & $2,7 \mathrm{a}$ \\
\hline Automatizado & $319 \mathrm{a}$ & $4,8 \mathrm{~b}$ & $24 \mathrm{a}$ & $203 b$ & $603 \mathrm{a}$ & $134 \mathrm{a}$ & $1,5 \mathrm{a}$ & $3,0 \mathrm{a}$ \\
\hline C. V. $(\%)$ & 16,83 & 25,16 & 15,88 & 29,65 & 10,49 & 17,46 & 18,81 & 52,10 \\
\hline
\end{tabular}

${ }^{(1)}$ Diâmetro da mediana volumétrica $(\mu \mathrm{m})$; (2) Coeficiente de homogeneidade; (3) Percentagem de cobertura; ${ }^{(4)}$ População de gotas (gotas cm $\left.{ }^{-2}\right)$; ${ }^{(5)}$ Amplitude relativa; ${ }^{(6)}$ Deposição de calda $\left(\mu \mathrm{L} \mathrm{cm}^{-2}\right)$. Médias seguidas de letras iguais, na coluna, não diferem entre si, pelo teste Tukey, a $5 \%$.

sua interferência nos parâmetros da pulverização. Observou-se, nesta fase do experimento, que apenas o coeficiente de homogeneidade $(\mathrm{CH})$ e a população de gotas (POP) diferiram entre os sistemas de pulverização empregados (Tabela 3 ).

O menor coeficiente de homogeneidade, obtido no sistema automatizado, indicou que, neste caso, a uniformidade das gotas foi melhor quando o sistema estava funcionando com o controle automático de pressão. O sistema automatizado apresentou coeficiente dentro do intervalo de 1,8 a 5,0, proposto por Marques (1994) e Teixeira (2010), e, no sistema manual, o coeficiente foi de 5,4. Já a população de gotas, para ambos os sistemas, foi superior aos valores propostos por Barthelemy et al. (1990), sendo consideradas satisfatórias para o controle de insetos e patógenos.

Os resultados obtidos são indicativos da boa aplicabilidade da tecnologia embarcada em máquinas e implementos agrícolas, sendo uma tecnologia cujo apelo técnico e comercial é grande. Outras razões que justificam a necessidade de pesquisas em automação e inovações na pulverização de agrotóxicos são o alto custo da mão de obra no campo, o risco de acidentes, a intoxicação de operadores e a redução na contaminação de alimentos e do meio ambiente. Entretanto, as pesquisas destinadas ao desenvolvimento de sistemas autônomos robustos, aplicáveis ao campo e capazes de se adaptarem à rotina de trabalho, nas propriedades agrícolas, além de realizarem múltiplas tarefas, ainda é bastante cara e, por isto, restrita. Entretanto, para Auernhammer \& Speckmann (2006), seguindo a tendência dos sistemas hidráulicos e elétricos, o uso de equipamentos eletrônicos e de computadores embarcados têm se difundido cada vez mais em máquinas agrícolas.

É importante destacar que a vantagem da automatização do pulverizador não foi, necessariamente, produzir uma aplicação com qualidade superior àquela conduzida manualmente. Além disto, um problema cada vez maior no campo é a escassez e o custo da mão de obra qualificada. Algumas das vantagens da automação são a sustentabilidade no processo produtivo e o fomento ao desenvolvimento econômico e social, corroborando os resultados obtidos por Menezes \& Martins (2009).

Uma alternativa bastante viável ao controle de vazão é o emprego de eletroválvulas de vazão proporcional, que apresentam tempo de resposta menor que o servomotor utilizado nesta pesquisa. A interface entre operador e sistema pode indicar as marchas de trabalho, porém, como a velocidade é variável, de acordo com o terreno, sempre há a necessidade de se realizar testes no campo, devido a restrições no trânsito da máquina. Esta tecnologia constitui-se, também, em uma alternativa para máquinas não tripuladas e tratores com sistema de piloto automático, dentre outros. A utilização de um sistema de ajuste do espectro de gotas, em função das condições meteorológicas, mantendo-se o volume de calda constante, por meio da mudança de velocidade, apresenta bastante potencial, uma vez associado à tecnologia de injeção direta de agrotóxicos.

\section{CONCLUSÕES}

1. O sistema automatizado proporcionou pulverização eficiente, considerando-se os parâmetros da aplicação de agrotóxicos estudados.

2. A automatização permitiu a manutenção do volume de calda, alterando a pressão de trabalho, em função do déficit de pressão de vapor d'água no ar.

3. A pulverização com sistema automatizado proporcionou qualidade do espectro de gotas superior ao sistema manual, à pressão de $633 \mathrm{kPa}$.

\section{REFERÊNCIAS}

ARDUINO. Arduino Duemilanove. 2011. Disponível em: $<\mathrm{http}$ ://www.arduino.cc/en/ArduinoBoardDuemilanove $>$. Acesso em: 17 maio 2011. 
AUERNHAMMER, H.; SPECKMANN, H. Dedicated communication systems and standards for agricultural applications. In: ASABE. CIGR handbook of agricultural engineering. St. Joseph: ASABE, 2006. p. 435-435.

BALAN, M. G. et al. Deposição da calda pulverizada por três pontas de pulverização sob diferentes condições meteorológicas. Semina: Ciências Agrárias, Londrina, v. 29, n. 2, p. 293-298, 2008.

BARTHELEMY, P. et al. Choisir les outilis de pulverisation. Paris: Institut Technique des Céréales et des Fourrages, 1990.

CÂMARA, F. T. et al. Distribuição volumétrica e espectro de gotas de bicos hidráulicos de jato plano de faixa expandida XR11003. Engenharia Agrícola, Jaboticabal, v. 28, n. 4, p. 740-749, 2008.

CUNHA, J. P. A. R.; BUENO, M. R.; FERREIRA, M. C. Espectro de gotas de pontas de pulverização com adjuvantes de uso agrícola. Planta Daninha, Viçosa, v. 28, n. esp., p. 1153-1158, 2010.

DÍAZ, S. E. et al. A novel methodology for the monitoring of the agricultural production process based on wireless sensor networks. Computers and Electronics in Agriculture, Amsterdam, v. 76, n. 2, p. 252-265, 2011.

GIL, Y. et al. Influence of micrometeorological factors on pesticide loss to the air during vine spraying: data analysis with statistical and fuzzy inference models. Biosystems Engineering, Columbus, v. 100, n. 2, p. 184-197, 2008.

JEON, H. Y. et al. Evaluation of ultrasonic sensor for variable-rate spray applications. Computers and Electronics in Agriculture, Amsterdam, v. 75, n. 1, p. 213$221,2011$.

LLORENS, J. et al. Variable rate dosing in precision viticulture: use of electronic devices to improve application efficiency. Crop Protection, Oxford, v. 29, n. 3, p. 239248, 2010.

MARQUES, L. Aplicaciones en cultivos bajos y hortícolas: problemática y soluciones: curso de aplicación ecocompatible de productos fitosanitarios. Madrid: Escuela Técnica Superior de Ingenieros Agrónomos, 1994.

MENEZES, D.; MARTINS, D. S. Automação e controle de pulverização em máquinas agrícolas. Revista de Graduação Univem, Marília, v. 1, n. 2, p. 23-34, 2009.
PEREIRA, R. R. D. Protocolo ISO 11783: procedimentos para comunicação serial de dados com o controlador de tarefas. 2008. 188 f. Dissertação (Mestrado em Engenharia Mecânica) - Escola de Engenharia de São Carlos, Universidade de São Paulo, São Carlos, 2008.

PEZZOPANE, J. R. M. et al. Condições microclimáticas em cultivo de café conilon a pleno sol e arborizado com nogueira macadâmia. Ciência Rural, Santa Maria, v. 40, n. 6 , p. 1257-1263, 2010.

SCARLETT, A. J. Integrated control of agricultural tractors and implements: a review of potential opportunities relating to cultivation and crop establishment machinery. Computers and Electronics in Agriculture, Amsterdam, v. 30, n. 1-3, p. 167-191, 2001.

SILVA, K. O. et al. Sistema automatizado para aquisição de dados de umidade relativa do ar. Engenharia Agrícola, Jaboticabal, v. 27, n. 3, p. 630-638, 2007.

TEIXEIRA, M. M. Estudio de la población de gotas de pulverización. In: TEIXEIRA, M. M. Tecnología de aplicación de agroquímicos. Alto Valle: INTA, 2010. cap. 5 .

TESKE, M. E.; THISTLE, H. W.; LONDERGAN, R. J. Modification of droplet evaporation in the simulation of fine droplet motion using AGDISP. Transactions of the $A S A B E$, St. Joseph, v. 54, n. 2, p. 417-421, 2011.

TETENS, V. O. Über einige meteorologische Begriffe. Zeitschrift Geophysic, Wurzburg, v. 6, n. 1, p. 297-309, 1930.

VAISALA. HMP50 miniature humidity and temperature probe for OEM applications. 2011. Disponível em: $<$ http:// br.vaisala.com/files/HMP50_Datasheet_in_English.pdf $>$. Acesso em: 13 maio 2011.

XU, L. et al. Evaporation rate and development of wetted area of water droplets with and without surfactant at different locations on waxy leaf surfaces. Biosystems Engeneering, Columbus, v. 106, n. 1, p. 58-67, 2010.

YU, Y. et al. Evaporation and deposition coverage area of droplets containing insecticides and spray additives on hydrophilic, hydrophobic, and crabapple leaf surfaces. Transactions of the ASABE, St. Joseph, v. 52, n. 1, p. 3949, 2009. 